\title{
Dysplasia Involves Enteritis Cystica Profunda in the Setting of Crohn's Disease
}

\author{
Jonathon Mahlow ${ }^{\mathrm{a}}$, Meagan Costedio ${ }^{\mathrm{b}}$, Shu-Yuan Xiao ${ }^{\mathrm{c}}$, Lisi Yuan ${ }^{\mathrm{a}}$, \\ Xiuli Liu ${ }^{\mathrm{a}, \mathrm{d}}$
}

\begin{abstract}
Enteritis cystica profunda is a rare non-neoplastic condition characterized by misplaced glands deep to the muscularis mucosae of the small bowel, likely as a result of trauma or inflammation such as Crohn's disease, a disease with increased risk for small bowel adenocarcinoma. Clinically, enteric cystica profunda and adenocarcinoma can present with obstruction and/or stricture in patients with Crohn's disease and should be differentiated from each other. Neoplastic involvement of enteritis cystica profunda by dysplasia has not been reported previously. Herein, we report a case of enteritis cystica profunda involved by high-grade dysplasia of gastric type. High-grade dysplasia and intramucosal adenocarcinoma of gastric type were also present in the overlying mucosa. Additionally, the adjacent mucosa demonstrated marked chronic active enteritis with pyloric gland metaplasia. The neoplastic epithelium showed diffuse and strong nuclear immunoreactivity for $\mathrm{p} 53$. The presence of lamina propria deep to the dysplastic epithelium-lined cysts/diverticula supported a non-invasive nature of this lesion. No metastatic disease was noted in 15 nodes identified. All surgical resections were free of neoplasm. The patient was doing well 9 months after surgery without evidence of cancer recurrence or metastasis on abdominal computed tomography.
\end{abstract}

Keywords: Enteritis cystica profunda; Crohn's disease; Dysplasia

\section{Introduction}

Crohn's disease, an idiopathic inflammatory bowel disease, often involves the small bowel. Chronic inflammation, mucosa injury, mucosa regeneration, and mural fibrosis may lead to en-

Manuscript accepted for publication January 11, 2016

aDepartment of Anatomic Pathology, Cleveland Clinic, Cleveland, OH, USA bepartment of Gastroenterology and Hepatology, Digestive Disease Institute, Cleveland Clinic, Cleveland, OH, USA

${ }^{\mathrm{c}}$ Department of Pathology, University of Chicago, IL, USA

${ }^{\mathrm{d} C}$ Corresponding Author: Xiuli Liu, Department of Anatomic Pathology, Cleveland Clinic, 9500 Euclid Avenue/L25, Cleveland, OH 44195, USA.

Email: liux3@ccf.org

doi: http://dx.doi.org/10.14740/jmc2415w teritis cystic profunda, a rare non-neoplastic condition characterized by misplaced glands deep to the muscularis mucosae of the small bowel which may cause stricture radiographically or obstruction clinically $[1,2]$. Chronic inflammation of mucosa (chronic enteritis) in Crohn's disease also increases the risk of small bowel adenocarcinoma via an inflammation-dysplasiaadenocarcinoma sequence [3-5]. Crohn's disease-associated small bowel adenocarcinomas have been reported to have an endometriosis-like invasion pattern and manifest gastric differentiation in the majority of cases $[6,7]$. This is further supported by positive immunohistochemistry staining for gastric markers, MUC5AC and MUC6 [7]. Dysplasia has been reported to be present in at least 50\% of Crohn's disease-associated small bowel adenocarcinoma $[4,5]$ and overexpression of $\mathrm{p} 53$ has been noted in $60 \%$ of Crohn's disease-associated dysplasia [5].

In patients with Crohn's disease, both enteritis cystica profunda and small bowel adenocarcinoma can occur and share similar clinical presentations, i.e., obstruction and/or stricture, thus they should be differentiated from each other. Recently, one case of colitis cystica profunda indefinite for dysplasia in Crohn's disease was reported as a potential diagnostic pitfall [8]. But neoplastic involvement of enteritis cystica profunda in Crohn's disease has not been reported in the literature. Herein, we report the first case of dysplasia involving enteritis cystica profunda in the setting of Crohn's disease. Our report highlights the diagnostic rarity and difficulty of this entity.

\section{Case Report}

A 68-year-old female with a long history of Crohn's disease presented to our hospital for further evaluation of abdominal pain of 1-year duration. She had an exploratory laparotomy in 1979 for a pelvic cyst and abdominal pain and was found to have Crohn's disease of the terminal ileum and underwent an appendectomy and isolated small bowel resection $(10-12 \mathrm{~cm})$ with handsewn end-to-end anastomosis sparing the ileocecal valve. After surgery, she felt well, and had an average of 3 - 4 bowel movements per day with minimal pain on no Crohn's therapy. She was not feeling well for some time, but did not seek medical attention until the year prior to surgery. She began having increased cramping abdominal pain and borborygmi and returned to the gastroenterologist. She underwent 


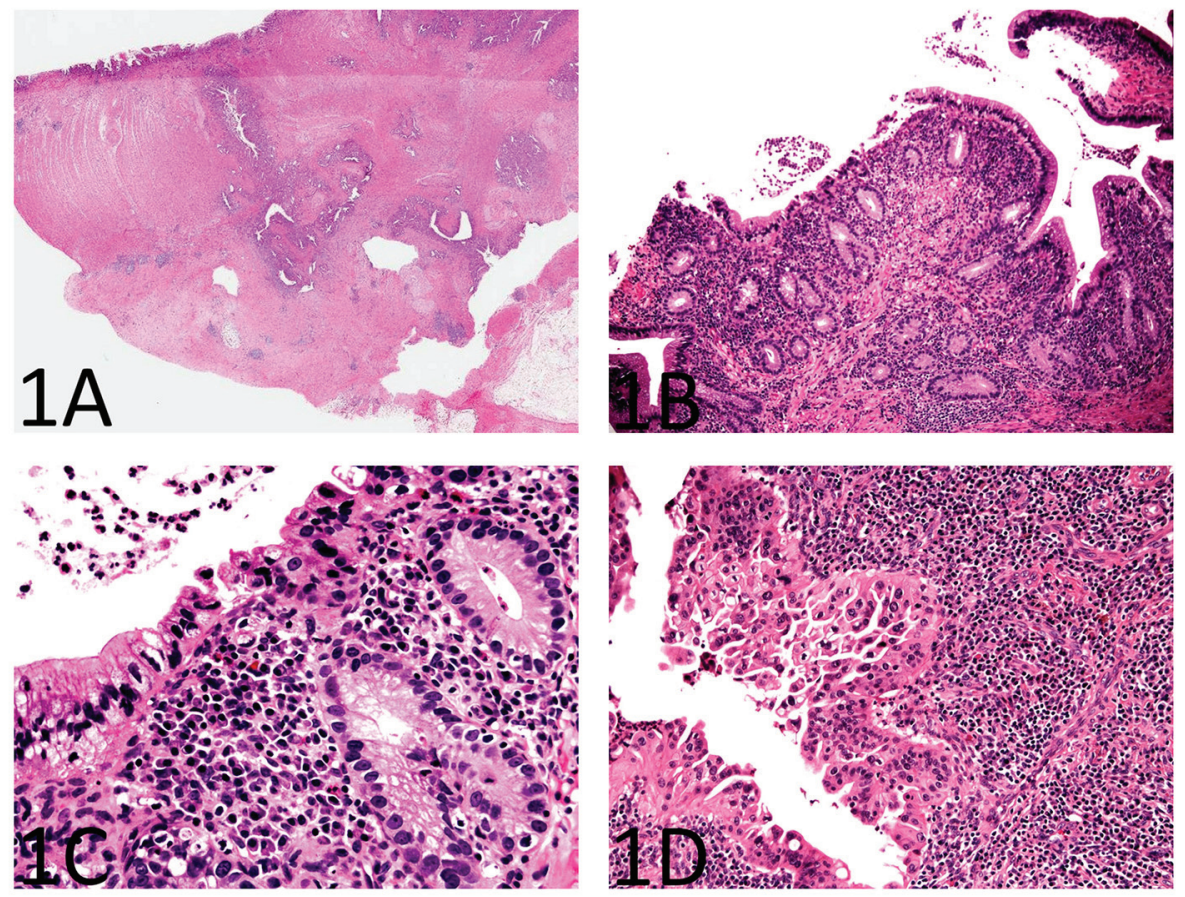

Figure 1. Histopathology of enteritis cystica profunda with high-grade dysplasia in the setting of Crohn's disease of the small bowel. (A) A section from the stricture shows chronic active enteritis with extensive ulceration, submucosal and mural fibrosis, hypertrophy of muscular wall, transmural inflammation including prominent lymphoid aggregates, and neural hypertrophy (H\&E, $\times 40$ ). There are many, variably sized, cysts or diverticulum predominantly lined by small bowel type mucosa. Some of the cysts are seen in the deep portion of the muscularis propria and in the subserosa. (B) The small bowel mucosa lining the cysts is chronically and actively inflamed and the epithelium resembling pyloric glands composed of cuboidal cells $(H \& E, \times 100)$. (C) The cyst-lining cuboidal cells are monotonous, have relatively high nucleus to cytoplasm (N/C) ratio, and do not show surface maturation, features of low-grade dysplasia (H\&E, $\times 400)$. (D) Some of the cyst-lining cuboidal cells have thickened nuclear membranes and demonstrate nuclear pleomorphism and loss of nuclear polarity, features of high-grade dysplasia (H\&E, $\times 400)$.

colonoscopy and was found to have a stricture at the anastomosis. She had occasional diarrhea, but overall continued having 4 - 5 bowel movements per day. She reportedly had lost some weight and weighed $100 \mathrm{lb}$ at the time of surgery. She had not tried Crohn's medications other than prednisone and budesonide in the past year. Budesonide helped with the bloating and pain but was too expensive. Lialda and azathioprine were not helpful in symptom relief in the past.

The patient's past medical history was remarkable for ovarian failure, thyrotoxicosis (Grave's disease, status post ablation), calculus of kidney (calcium oxylate), osteoporosis, benign essential hypertension, cholelithiasis, and thrombocytosis. The patient's social history was remarkable for being a former smoker (20 pack years), quit 17 years ago.

On physical examination, she was very thin, but appeared well nourished. Her abdomen was soft and flat without distension. There was right lower quadrant tenderness without rebound or rigidity. No hepatomegaly or masses were noted. Her bowel sounds were normal. Laboratory tests showed slight hypoalbumin at $3.4 \mathrm{~g} / \mathrm{dL}$, slightly low total protein at $5.9 \mathrm{~g} / \mathrm{dL}$,
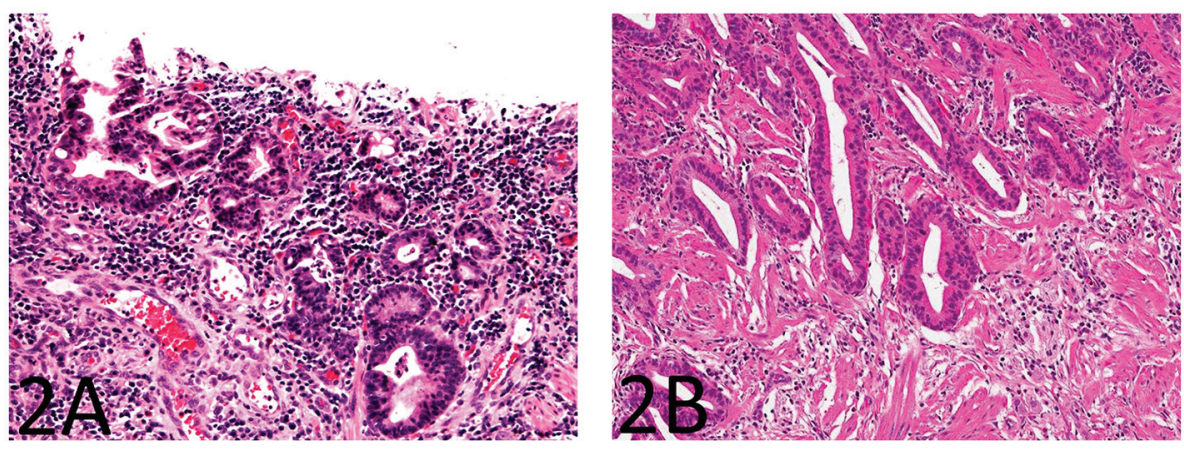

Figure 2. The small bowel mucosa overlying the strictured segment shows marked chronic active enteritis with high-grade dysplasia and intramucosal adenocarcinoma of gastric type (A: H\&E stain, $\times 200 ; B: H \& E$ stain, $\times 100$ ). 

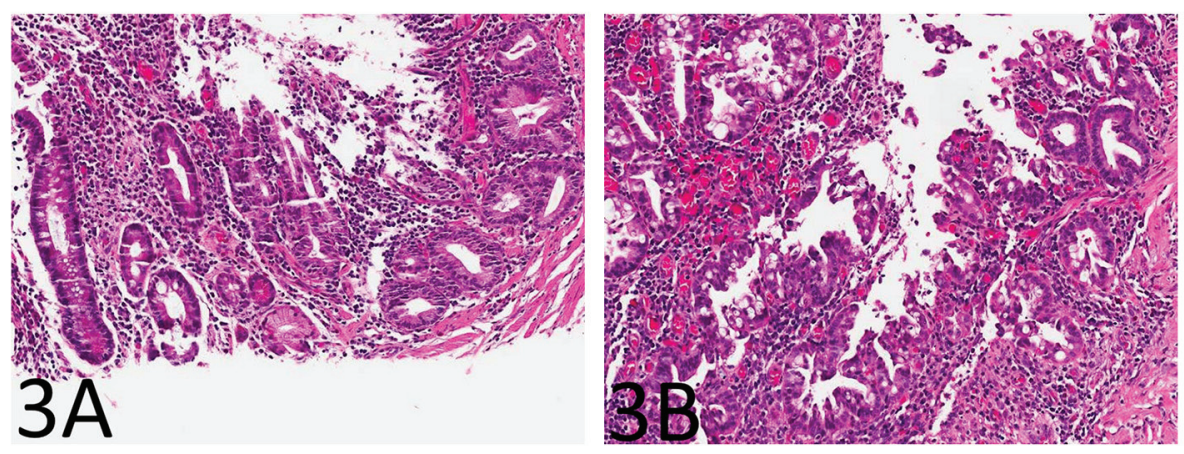

Figure 3. The small bowel mucosa from the area adjacent to the stricture shows chronic active enteritis with focal pyloric gland metaplasia (A: H\&E stain, $\times 100)$ and areas of low-grade dysplasia and high-grade dysplasia resembling the epithelial lining of the enteritis cystic profunda (B: H\&E stain, $\times 100)$.

normal ALT at $16 \mathrm{U} / \mathrm{L}$ and AST at $28 \mathrm{U} / \mathrm{L}$, and normal total bilirubin at $0.4 \mathrm{mg} / \mathrm{dL}$. Her hemoglobin was normal at $12.1 \mathrm{~g} /$ $\mathrm{dL}$ and white cell count (WBC) was at 5,260/ $\mu \mathrm{L}$.

Colonoscopy did not reveal Crohn's disease in the colon; however, terminal ileal intubation was not possible. Computed tomography (CT) enterography with oral contrast showed dilated proximal small bowel with fecalization and air fluid levels with a $10 \mathrm{~cm}$ edematous and thick walled stricture of the terminal ileum. Inflammation of the fat adjacent to the terminal ileum and enlarged ileocecal lymph node were also present. There was neither abscess nor pneumoperitoneum. There was no colonic thickening or pericolonic inflammation.

The patient underwent a single incision laparoscopic ileocolic resection. There were few adhesions from her prior surgery, and the terminal ileal disease was easily identified and dissected from the retroperitoneum. The small bowel was run and significant strictures at the prior anastomosis site as well as $10 \mathrm{~cm}$ proximal to that were identified. The remainder of the small bowel and colon appeared normal. An end to side stapled anastomosis was performed. The mesentery was taken close to the bowel wall to avoid mesenteric hematoma in the inflamed Crohn's mesentery.

\section{Macroscopic findings}

The specimen consisted of a segment of ileum and colon measuring $27.0 \mathrm{~cm}$ in length. There was mild fibrous adhesion on the serosa of the small bowel. The serosal aspect of the colon was smooth and glistening. An area of stricture was present between the segment of ileum and colon and the bowel circumference at this point measured $2.0 \mathrm{~cm}$. Proximally, the bowel appeared dilated measuring up to $12.0 \mathrm{~cm}$ in circumference. No areas of induration, hemorrhage, or abscess cavities were identified. The mucosal surface particularly within the area of the stricture demonstrated areas of ulceration and hemorrhage. No masses or lesions were identified macroscopically. Polyps were not present. The muscularis propria appeared thickened, particularly within the area of the stricture, measuring up to $0.7 \mathrm{~cm}$ in thickness. The colon was macroscopically unremarkable. The stricture segment was entirely submitted for histo- logically examination.

\section{Microscopic findings}

As shown in Figure 1A, sections from the stricture showed chronic active enteritis with extensive ulceration, submucosal and mural fibrosis, hypertrophy of muscular wall, transmural inflammation including prominent lymphoid aggregates, neural hypertrophy, and fibrous adhesions. Most remarkably, there were many, variably sized, cysts or diverticulum predominantly lined by small bowel type mucosa. Some of the cysts were seen in the deep portion of the muscularis propria and in the subserosa (Fig. 1A). Rare cysts were partially ulcerated and lined by granulation tissue.

The small bowel mucosa lining the cysts was chronically and actively inflamed and the epithelium resembling pyloric glands was composed of cuboidal cells. However, these cuboidal cells were monotonous, had relatively high nucleus to cytoplasm (N/C) ratio, and did not show surface maturation (Fig. 1B). Some of the cells had thickened nuclear membranes and a single prominent nucleolus. Focal nuclear pleomorphism and loss of nuclear polarity were also noted (Fig. 1C, D). Mitotic figures were readily identifiable. The mucosa overlying the strictured segment showed marked chronic active enteritis with high-grade dysplasia and intramucosal adenocarcinoma of gastric type (Fig. 2A, B). The mucosa from the area adjacent to the stricture showed chronic active enteritis with focal pyloric gland metaplasia (Fig. 3A) and areas of low-grade dysplasia and high-grade dysplasia resembling the epithelial lining of the enteritis cystic profunda (Fig. 3B). In addition, intramucosal adenocarcinoma was also noted in the adjacent surface mucosa (not shown). No granulomas were identified.

The antibodies used for immunohistochemical analysis were shown in Table 1.

Immunohistochemistry for $\mathrm{p} 53$ showed diffuse and strong nuclear immunoreactivity in the epithelium of the enteritis cystica profunda (Fig. 4A, B) as well as its overlying mucosa (Fig. 4C) and adjacent mucosa, supporting the neoplastic nature of the epithelium. As shown in Figure 5A-F, the lesional epithelium showed strong and diffuse immunoreactivity for gastric markers, MUC5AC and MUC6 in addition to CK7, but 
Table 1. Antibodies Used for Immunohistochemical Analysis

\begin{tabular}{llll}
\hline Antigen & Clone/antibody & Dilution & Source \\
\hline p53 & $\begin{array}{l}\text { Clone: DO-7 } \\
\text { Catalog no.: M7001 }\end{array}$ & $1: 20$ & Dako \\
Cytokeratin 7 & $\begin{array}{l}\text { Clone: OV-TL 12/30 } \\
\text { Catalog no.: M7018 }\end{array}$ & $1: 40$ & Dako \\
Cytokeratin 20 & $\begin{array}{l}\text { Clone: Ks20.8 } \\
\text { Catalog no.: M7019 }\end{array}$ & $1: 20$ & Dako \\
CDX2 & Clone: CDX2-88 & $1: 100$ & Biogenex \\
MUC2 & CCP58 & $1: 25$ & NovoCastra, UK \\
MUC5AC & CLH2 & $1: 25$ & NovoCastra, UK \\
MUC6 & CLH5 & $1: 25$ & NovoCastra, UK \\
\hline
\end{tabular}

only weak and focal immunoreactivity for intestinal marker CDX2 or colonic markers MUC2 and CK20 (Fig. 5A-F).

The patient had been doing well without evidence of recurrent cancer or metastasis at 9 months after surgery.

\section{Discussion}

Small bowel adenocarcinoma is an important and potentially fatal complication of long-term Crohn's disease of the small bowel and about $60 \%$ of these patients present with small bowel obstruction [9]. Similarly, enteritis cystica profunda can occur as a rare complication of Crohn's disease, clinically and radiographically mimicking Crohn's disease-associated ade- nocarcinoma. Histologically, both are characterized by glands infiltrating beyond the muscularis mucosae [1, 2, 10-12]. Differentiation of these two entities is usually straightforward in most cases but can be challenging in rare cases. Dysplasia involving enteritis cystica profunda has not been reported, and would potentially complicate the diagnosis.

One case of colitis cystica profunda indefinite for dysplasia in Crohn's disease had been reported previously [8]. In this case report, the authors reported the presence of a stricture associated with a multilocular cystic lesion containing colonic-wall dissecting mucin-filled glands which had lobulated architecture and were invested by lamina propria. However, the glandular epithelium within this cystic lesion showed focal nuclear crowding, enlargement, and hyperchromasia, with

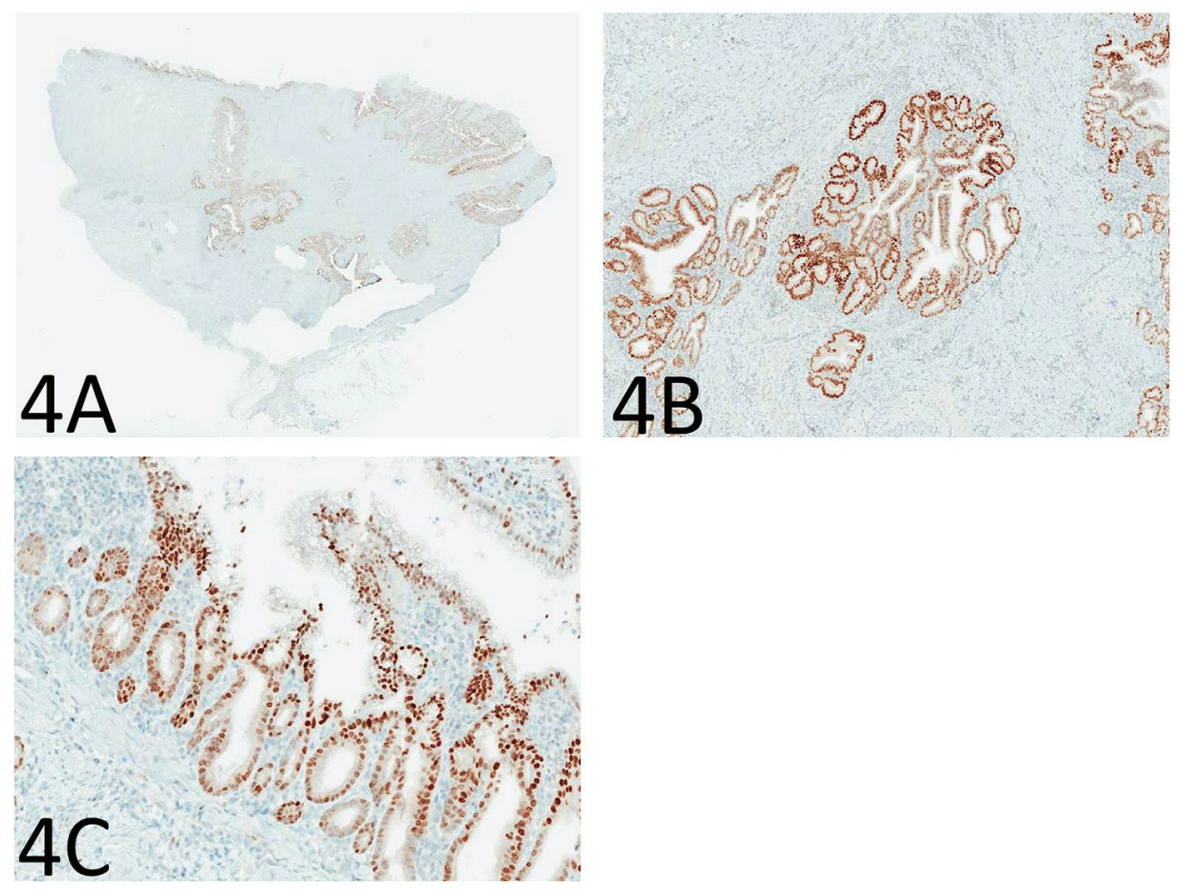

Figure 4. Immunohistochemistry for p53 shows diffuse and strong nuclear immunoreactivity in the epithelium of the enteritis cystica profunda (A: immunoperoxidase stain, $\times 40$; B: immunoperoxidase stain, $\times 100)$ as well as its overlying mucosa $(C$ : immunoperoxidase stain, $\times 100)$, supporting the neoplastic nature of the epithelium. 

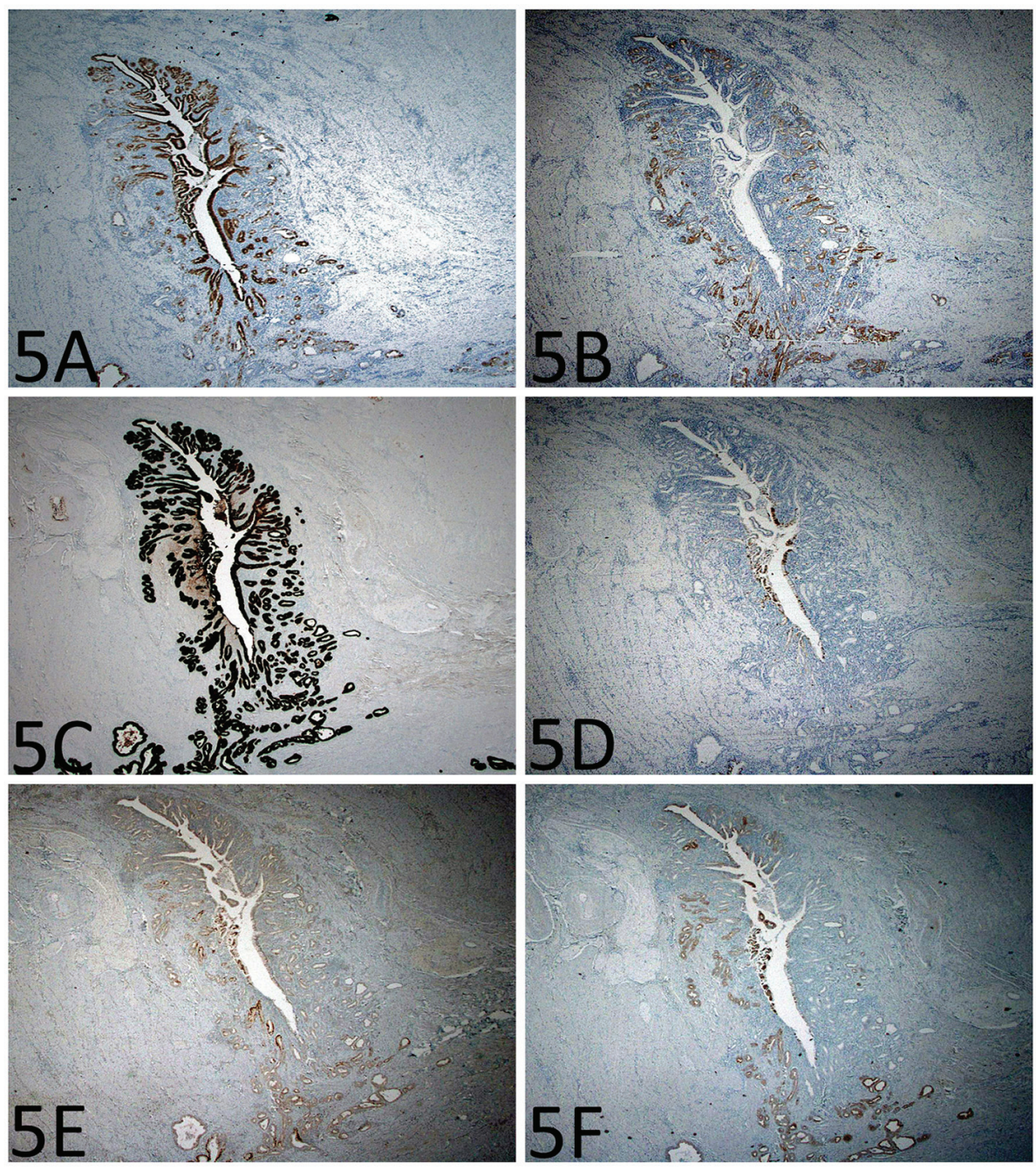

Figure 5. Immunohistochemistry shows gastric differentiation in the epithelium of the enteritis cystica profunda. (A) MUC5AC immunoreactivity in the foveolar type epithelium (immunoperoxidase stain, $\times 200$ ). (B) MUC6 immunoreactivity in the mucus glands (immunoperoxidase stain, $\times 200$ ). (C-F) There is strong CK7 immunoreactivity, but only focal CDX2, MUC2, and CK20 immunoreactivity (immunoperoxidase stain, $\times 200$ ).

increased N/C ratio, but with largely preserved polarity. By immunohistochemistry, this atypical glandular epithelium showed focal positivity for CK7 and p53 with increased MIB1 staining. The case report was reported as colitis cystica profunda indefinite for dysplasia in Crohn's disease. Although one case of intramucosal adenocarcinoma involvement of diverticula in Crohn's disease has been reported, enteritis cystica profunda with dysplasia has not been reported [3].

Here we present the first case of enteritis cystic profunda with dysplasia in a patient with long-term Crohn's disease as an unexpected diagnosis. Clinically, the patient was thought to have a benign stricture due to Crohn's disease. Grossly, the involved segment showed stricture and thickening of small bowel but without obvious cysts or mucin pools. However, sections through the stricture clearly showed cysts and diverticula lined by chronically and actively inflamed small intestinal type mucosa including appreciable lamina propria in the muscularis propria and subserosa. The overall configuration of the cysts and diverticula was round and lobular. In addition, the cysts and diverticulae-lining epithelium showed uniform cuboid cells with high-grade dysplasia of gastric differentiation, both histologically and immunophenotypically. The presence of dysplasia and intramucosal adenocarcinoma of gastric type in the overlying mucosa and the adjacent mucosa further supported the interpretation of neoplastic involvement of enteritis cystic profunda in this case. The neoplastic/dysplastic nature of this lesion was further supported by the diffuse nuclear immunoreactivity for $\mathrm{p} 53$, which had been reported previously in Crohn's enteritis-associated dysplasia/adenocarcinoma [5, 13]. The major differential diagnosis for this case included a deeply invasive, well-differentiated, adenocarcinoma (the socalled "intestinal low-grade tubuloglandular adenocarcinoma" in inflammatory bowel disease [14] and an endometriosis-like adenocarcinoma) [6]. Importantly, the lobular contour of the lesion and the presence of lamina propria in these deeply situated cysts/diverticula provide reassurance that this is not an 
invasive adenocarcinoma.

It can be argued that this case may not represent an enteritis cystica profunda, commonly described as cysts lined by mucinous epithelium with or without mucin pool. However, the current case had many variably sized cysts deeply situated in the muscularis propria and subserosa. These findings fit a broad definition of enteritis cystica profunda, i.e., the presence of misplaced glands deep to the muscularis mucosae of the small bowel and yet still lined by lamina propria. The lack of mucin may be due to the neoplastic replacement of the cyst lining epithelium which may have less mucin-secreting capacity.

This case shows several points of interest. First, this highgrade dysplasia and intramucosal adenocarcinoma occurred in a patient of an older rather than young age. The mean age at onset of Crohn's disease-associated small bowel adenocarcinoma in one report is 48 years [5]. Second, this neoplastic process extensively involves the enteritis cystica profunda. Third, the neoplastic epithelium shows marked gastric differentiation, both histomorphologically and immunophenotypically. This finding is consistent with previous observations and reports that Crohn enteritis-associated small bowel dysplasia and adenocarcinomas tend to exhibit gastric differentiation [4, 7]. The lobular contour of the lesion and the presence of lamina propria in the deeply situated cysts and diverticula helped rule out invasive adenocarcinoma. In addition, a finding of pyloric gland metaplasia, dysplasia of gastric type, and intramucosal adenocarcinoma (in the mucosa overlying and adjacent to the enteritis cystica profunda) supports a gastric metaplasia-carcinoma hypothesis in Crohn's disease-associated small bowel tumorigenesis, as suggested in previous case reports or series $[4,7,15,16]$. Finally, an awareness of this unique form of small intestinal dysplasia, i.e., gastric type, may help avoid underdiagnosing this as non-neoplastic epithelium on frozen sections when the surgeon submits tissue from the strictured small bowel segment before performing strictureplasty, an alternative management option for some Crohn's patients with stricture.

In summary, we report the first case of enteritis cystica profunda involved by high-grade dysplasia of gastric type in one patient with Crohn's disease presenting with small bowel obstruction. Adequate sampling of the strictured area and the adjacent mucosa as well as an awareness of this rare entity is essential for the correct diagnosis. Attention to the overall configuration and the composition of the deeply situated cysts and diverticula help avoid misdiagnosing it as a deeply invasive adenocarcinoma.

\section{References}

1. Saul SH, Wong LK, Zinsser KR. Enteritis cystica profunda: association with Crohn's disease. Hum Pathol. 1986;17(6):600-603.

2. Alexis J, Lubin J, Wallack M. Enteritis cystica profunda in a patient with Crohn's disease. Arch Pathol Lab Med. 1989;113(8):947-949.

3. Perzin KH, Peterson M, Castiglione CL, Fenoglio CM, Wolff M. Intramucosal carcinoma of the small intestine arising in regional enteritis (Crohn's disease). Report of a case studied for carcinoembryonic antigen and review of the literature. Cancer. 1984;54(1):151-162.

4. Simpson S, Traube J, Riddell RH. The histologic appearance of dysplasia (precarcinomatous change) in Crohn's disease of the small and large intestine. Gastroenterology. 1981;81(3):492-501.

5. Svrcek M, Piton G, Cosnes J, Beaugerie L, Vermeire S, Geboes K, et al. Small bowel adenocarcinomas complicating Crohn's' disease are associated with dysplasia: a pathologic and molecular study. Inflamm Bowel Dis. 2014;20:1854-1592.

6. Fleming KA, Pollock AC. A case of 'Crohn's carcinoma'. Gut. 1975;16(7):533-537.

7. Whitcomb E, Liu X, Xiao SY. Crohn enteritis-associated small bowel adenocarcinomas exhibit gastric differentiation. Hum Pathol. 2014;45(2):359-367.

8. Hernandez-Prera JC, Polydorides AD. Colitis cystica profunda indefinite for dysplasia in Crohn disease: a potential diagnostic pitfall. Pathol Res Pract. 2014;210(12):10751078.

9. Elriz K, Carrat F, Carbonnel F, Marthey L, Bouvier AM, Beaugerie L. Incidence, presentation, and prognosis of small bowel adenocarcinoma in patients with small bowel Crohn's disease: a prospective observational study. Inflamm Bowel Dis. 2013;19(9):1823-1826.

10. Aftalion B, Lipper S. Enteritis cystica profunda associated with Crohn's disease. Arch Pathol Lab Med. 1984;108(7):532-533.

11. del Rey-Moreno A, Jimenez-Martin JJ, Moreno-Ruiz FJ, Hierro-Martin I. [A combination of enteritis cystica profunda, Crohn's ileocolitis and endometriosis as a cause of intestinal obstruction]. Cir Esp. 2008;83(5):271.

12. Dikinis S, Bohme WP. [Enteritis cystica profunda in a patient with Crohn disease]. Ugeskr Laeger. 2001;163(35):4755-4756.

13. Uesugi H, Mitomi H, Sada M, Takahashi H, Kobayashi K, Igarashi M, Katsumata T, et al. A case of adenocarcinoma of the small intestine in a Japanese patient with Crohn disease: a report with immunohistochemical and oncogenic analyses. Scand J Gastroenterol. 1999;34(11):1162-1167.

14. Levi GS, Harpaz N. Intestinal low-grade tubuloglandular adenocarcinoma in inflammatory bowel disease. Am J Surg Pathol. 2006;30(8):1022-1029.

15. Bearzi I, Ranaldi R. Small bowel adenocarcinoma and Crohn's disease: report of a case with differing histogenetic patterns. Histopathology. 1985;9(3):345-357.

16. Marchetti F, Fazio VW, Ozuner G. Adenocarcinoma arising from a strictureplasty site in Crohn's disease. Report of a case. Dis Colon Rectum. 1996;39(11):1315-1321. 